\title{
Geodesics without Conjugate Points and Curvatures at Infinity
}

\author{
SÉRGIO MENDONÇA and DETANG ZHOU \\ Universidade Federal Fluminense - UFF, Instituto de Matemática, \\ Campus do Valonginho, rua Mário Santos Braga s/n, $7^{\circ}$ andar - 24020-140 Niterói, RJ \\ Manuscript received on July 16, 1999; accepted for publication on February 8, 2000; \\ presented by MANFREDO DO CARMO
}

\begin{abstract}
We study the asymptotic behavior of curvature and prove that the integral of curvature along a geodesic without conjugate points is nonpositive and some generalizations of Myers theorem and Cohn-Vossen's theorem. Some applications are also given.
\end{abstract}

Key words: Riemannian manifold, geodesic, conjugate point.

\section{MAIN RESULTS}

Let $\mathbf{M}^{\mathbf{n}}$ be an $n$-dimensional Riemannian manifold and let $d(x, y)$ be the distance induced by the metric. (Ambrose 1957) showed that if the integral of the Ricci curvature along a geodesic $\gamma:[0,+\infty) \rightarrow M$ is infinite then there is a $t>0$ such that $\gamma(t)$ is conjugate to $\gamma(0)$. We extended this result in two directions: first we obtain

THEOREM A. Let $\gamma: \mathbb{R} \rightarrow M$ be a geodesic without conjugate points (particularly if $\gamma$ is a line). Then for any unit vector field $X(t)$ which is parallel along $\gamma$ it holds that

$$
\eta=\liminf _{\substack{u \rightarrow-\infty \\ v \rightarrow+\infty}} \int_{u}^{v} K\left(X(t), \gamma^{\prime}(t)\right) d t \leq 0, \quad \mu=\liminf _{\substack{u \rightarrow-\infty \\ v \rightarrow+\infty}} \int_{u}^{v} \operatorname{Ric}\left(\gamma^{\prime}(t), \gamma^{\prime}(t)\right) d t \leq 0
$$

where $K\left(X(t), \gamma^{\prime}(t)\right)$ is the sectional curvature of the plane spanned by $X(t)$ and $\gamma^{\prime}(t)$. Moreover, if $\eta=0$ then $K\left(X(t), \gamma^{\prime}(t)\right) \equiv 0$. If $\mu=0$ then for all $t$ and any $v$ orthogonal to $\gamma^{\prime}(t)$, it holds that $\left.K\left(\gamma^{\prime}(t), v\right)\right)=0$.

Note that $M$ is not supposed to be complete in Theorem A, and no hypothesis on the curvature is assumed. It should be remarked that (Liang \& Zhan 1996) proved that if $\gamma: \mathbb{R} \rightarrow M$ is a geodesic without conjugate points and if $\operatorname{Ric}\left(\gamma^{\prime}(t), \gamma^{\prime}(t)\right) \geq 0$, then $\operatorname{Ric}\left(\gamma^{\prime}(t), \gamma^{\prime}(t)\right) \equiv 0$. 
Recall that the minimal radial curvature $K_{o}^{\min } \geq c$ if $K\left(\gamma^{\prime}, v\right) \geq c$ for any minimal normal geodesic $\gamma$ joining $o$ and $p$ and any unit vector $v$ orthogonal to $\gamma^{\prime}$. We say that the radial Ricci curvature $\operatorname{Ric}_{o}(p) \geq c$ if for any normal (not necessarily minimizing) geodesic $\gamma$ joining $o$ and $p$ it holds that $\operatorname{Ric}\left(\gamma^{\prime}, \gamma^{\prime}\right) \geq c$. As a corollary of Theorem A we have

Corollary 1. Assume that $M$ is a complete manifold without conjugate points satisfying $\operatorname{Ric}_{o} \geq 0$, for a certain base point $o$. Then $M$ is flat, that is, $M$ is isometric to $\mathbb{R}^{n} / \pi_{1}(M)$.

It should be remarked that when $M$ is compact and the condition $\mathrm{Ric}_{o} \geq 0$ is replaced by the scalar curvature $S \geq 0$, the same conclusion of Corollary 1 follows from (Green 1958).

In order to state our second result we need some definitions. In the following $M=M^{n}$ always denotes a complete and connected $n$-dimensional Riemannian manifold. $S$ is always a connected manifold without boundary which is isometrically immersed and whose image is closed in $M$. This assumption is weaker than that the immersion being proper, since if $N$ is any compact manifold and $p \in N$ has no conjugate points, then the exponential map exp: $T_{p} N \rightarrow N$ is an immersion whose image is closed, but the inverse image of $N$ of course is not compact. Unless otherwise stated all geodesics are supposed to be normalized. One of the subjects treated here is to study the manifolds with minimal $S$-radial (Ricci) curvature bounded from below (or from above). Even if we strengthen our curvature conditions with the corresponding ones on the sectional (or Ricci) curvature some of our results are new.

The notion of minimal radial curvature was first introduced by (Klingenberg 1963) and was studied by many authors. It is natural to extend such definition for submanifolds when we study existence of minimal submanifolds. The notion of minimal $S$-radial curvature appears - even without an explicit definition - for example in (Eschenburg 1987) and in (Heintze \& Karcher 1978). Given $p \in M$ we say that a minimal geodesic $\gamma:[0, a] \rightarrow M$ is a minimal connection between $p$ and $S$ if $\gamma(0) \in S, \gamma(a)=p$ and the distance $d(p, S)=a$. Given linearly independent tangent vectors $v, w$ we denote by $K(v, w)$ the sectional curvature associated with the plane generated by $v$ and $w$.

0.1. Definition. Given $p \in M$, we say that the minimal $S$-radial curvature $K_{S}^{\min }(p) \geq c$ $\left(K_{S}^{\min }(p) \leq c\right)$ if for any minimal connection $\gamma$ between $p$ and $S$, and any $v$ orthogonal to the tangent vector $\gamma^{\prime}$ at $p$ it holds that $K\left(\gamma^{\prime}, v\right) \geq c\left(K\left(\gamma^{\prime}, v\right) \leq c\right)$. We say similarly that the minimal $S$-radial Ricci curvature $\operatorname{Ric}_{S}^{\min }(p) \geq c$ if any orthonormal frame $e_{1}, e_{2}, \ldots, e_{n-1}$ which is orthogonal to $\gamma$ at $p$ satisfies $\sum_{i} K\left(\gamma^{\prime}, e_{i}\right) \geq c$. Finally we say that the parallel minimal $S$-radial Ricci curvature $\mathcal{R} \mathcal{C}_{S}^{\min }(p) \geq c$ if $\sum_{i=1}^{k} K\left(\gamma^{\prime}, e_{i}\right) \geq c$, where $e_{1}, e_{2}, \cdots, e_{k}$ are obtained by the parallel transport along $\gamma$ of an orthonormal basis of $T_{\gamma(0)} S$.

Note that if the dimension of $S$ is $n-1$ then $\operatorname{Ric}_{S}^{\min }(p) \geq c$ is equivalent to $\operatorname{RIC}_{S}^{\min }(p) \geq c$. We can give some examples of radial curvature bounded from below. One of the most well-known results relating the curvature and topology of a complete Riemannian manifold $M$ is the classical Theorem of (Myers 1941) which states that if the Ricci curvature with respect to unitary vectors 
in $M$ has a positive lower bound then $M$ is compact. For the distance function $\rho(\mathbf{x})=d(x, S)$ set $\mathbf{R}_{\mathbf{S}}=\mathbf{R}_{\mathbf{S}}(\mathbf{M}):=\sup \{\rho(\mathbf{x}) \mid \mathbf{x} \in \mathbf{M}\}$. The following result shows that, if $R_{S}=+\infty$, then the existence of a minimal submanifold implies that radial curvatures tend to be nonpositive in some integral sense. Precisely we have:

THeOREм B. Let $S$ be minimal and have dimension $k \geq 1$. Assume that $M$ satisfies $\mathcal{R I C}_{S}^{\min }(x) \geq$ $k K(\rho(x))$ for any $x \in M$, where $K(\rho)$ is supposed to be a continuous function. Then the condition $R_{S}=+\infty$ implies that liminf ${ }_{t \rightarrow+\infty} \int_{0}^{t} K(\rho) d \rho \leq 0$. Furthermore, if $\liminf _{t \rightarrow+\infty} \int_{0}^{t} K(\rho) d \rho=0$ then we have $K(\rho)=0$, for all $\rho$.

We remark that $R_{S}$ can be finite even if $M$ is noncompact. For example, let $S$ be a line in a cylinder $M$. When $\mathcal{R} \mathcal{I} C_{S}^{\min } \geq 0$ Theorem B implies that, if $K(\rho)$ as above is nonnegative, then $K(\rho) \equiv 0$. This fact however does not imply that $\mathcal{R} \mathcal{L} C_{S}^{\min }(x) \equiv 0$. It means only that for any $\rho>0$ it holds that $\inf \left\{\mathcal{R} \mathcal{I} C_{S}^{\min }(x) \mid \rho(x)=\rho\right\}=0$ (see for example the case in that $S$ is a meridian of a paraboloid).

If a ray $\gamma:[0,+\infty) \rightarrow M$ satisfies $\gamma(0) \in S$ and $\rho \circ \gamma(t) \equiv t$ we will say that $\gamma$ is an S-ray. By the same proof as in Theorem A we obtain the following result.

Corollary 2. Assume that $S$ is totally geodesic (respectively, minimal), has dimension $k \geq 1$, and that $\gamma$ is an $S$-ray. Let $v=v(t)$ be a parallel field along $\gamma$ with $v(0) \in T_{\gamma(0)} S$. Then we have $\liminf _{t \rightarrow+\infty} \int_{0}^{t} K\left(v, \gamma^{\prime}\right) d s \leq 0$ (respectively, $\left.\liminf _{t \rightarrow+\infty} \int_{0}^{t} \mathcal{R} \mathcal{L} C_{S}^{\min }\left(\gamma^{\prime}, \gamma^{\prime}\right) d s \leq 0\right)$. If this integral limit vanishes then $K\left(v, \gamma^{\prime}\right) \equiv 0$ (respectively, $\left.\mathcal{R} \mathcal{I} C_{S}^{\min }(\gamma(t)) \equiv 0\right)$.

The Theorems of (Cohn-Vossen 1935) and (Huber 1957) assert that if $n=2$ and the negative part of its Gaussian curvature is integrable, then $\int_{M} K d M \leq 2 \pi \chi(M)$, where $d M$ is the volume element of $M$, and $\chi(M)$ is the Euler characteristic of $M$. This implies that for this type of manifolds there does not exist a sequence of points $q_{k} \rightarrow \infty$ with $K \geq \delta$ in the ball $B_{q_{k}}\left(r_{k}\right)$ with center $q_{k}$, radius $r_{k}$, and volume $V$, for fixed positive numbers $\delta$ and $V$. It should be noted that this is false without the assumption on the integrability of the curvature. This can be seen if we consider $R^{2}$ with the periodic metric induced by the universal covering of a nonflat metric on torus. It has been asked by many mathematicians about the extension of theorems of Cohn-Vossen and Huber to the higher dimensions (see for example Yau 1991).

As pointed out before we first need some suitable integrability conditions about curvatures. It seems for us that the asymptotically nonnegative condition studied extensively by (Abresch 1985) is one reasonable choice. In dimension $n$ let $\mathbf{K}(\mathbf{p})$ be the infimum of the sectional curvatures at the point $p$. It would be interesting to obtain an integral inequality for the function $K$. However we have only obtained the nonexistence of a sequence as above in this case. We recall (see Abresch 1985) that the curvature of a complete manifold $M$ is asymptotically nonnegative if there exists a nonincreasing function $\kappa:[0,+\infty) \rightarrow[0,+\infty)$ such that $\int_{0}^{+\infty} t \kappa(t) d t<+\infty$, and $K(x) \geq-\kappa(d(o, x))$, for a fixed point $o$. Abresch obtained (Abresch 1985) a version of Toponogov Theorem for this class of manifolds, and Kasue constructed in (Kasue 1988) a compactification of such manifolds $M$. Our 
third theorem is the following.

THEOREM C. Let $M$ be a complete manifold with asymptotically nonnegative curvature. Take a sequence $q_{k} \rightarrow \infty$. Suppose that $K \geq \delta_{k}>0$ in $B_{q_{k}}\left(r_{k}\right)$, with $r_{k} \leq R$, where $R$ is a fixed number. Then $r_{k}^{2} \delta_{k} \rightarrow 0$.

In particular it is not possible that $K \geq \delta>0$ in $B_{q_{k}}\left(r_{k}\right)$, if the volume of $B_{q_{k}}\left(r_{k}\right)$ is a constant $V$. In fact, let $K_{0}$ be the minimum of $K(p)$ in $M$. Because of the Bishop-Gromov comparison volume Theorem we would have in that case $r_{k} \geq r_{V}$, where $r_{V}$ is the radius of a ball of volume $V$ in the hyperbolic space of constant curvature $K_{0}$. So we would not have $r_{k} \rightarrow 0$, and this contradicts Theorem $\mathrm{C}$. We can give an example to show that the condition $r_{k} \leq R$ is essential in Theorem $\mathrm{C}$, even if $K \geq 0$.

0.2. Definition. An embedded submanifold $S$ is said to be a polar submanifold if the normal exponential map $\exp ^{\perp}: v(S) \rightarrow M$ is a diffeomorphism.

0.3. Definition. $S$ is called a geometric soul if the distance function $\rho$ is convex.

Clearly any geometric soul is totally convex (any geodesic joining two points of $S$ is contained in $S$ ). In (Cheeger \& Gromoll 1972) it is proved that any totally convex set $C$ is of the form $C=N \cup \partial C$, where $N=N^{k}$ is a $C^{\infty}$ embedded submanifold and $\partial C$ is a boundary of $C^{0}$ class. Since our $S$ has no boundary we conclude that any geometric soul is of $C^{\infty}$ class and totally geodesic. For the case of nonnegative $S$-radial curvature we have the following result.

Theorem D. Assume that $S$ is a $C^{2}$ polar submanifold of $M$ and that $K_{S}^{\min } \geq 0$. Given $x \in M \backslash S$ and a unitary vector $v \in T_{x} M$ it holds that the Hessian

$$
0 \leq \operatorname{Hess}_{\rho}(v, v) \leq \frac{1}{\rho(x)},
$$

hence the function $\rho$ is convex and $S$ is a geometric soul. In fact even if $S$ is not polar the conclusion $\operatorname{Hess}_{\rho}(v, v) \leq \frac{1}{\rho(x)}$ is valid in all point $x$ outside the cut locus of $S$.

Using Theorem D we can prove:

Corollary 3. Let $M$ satisfy $K_{S}^{\min } \geq 0$ (or $K_{S}^{\mathrm{min}} \leq 0$ and $S$ be totally geodesic). Then the distance function $d(\cdot, S)$ is convex if and only if $S$ is polar. In particular, under these conditions the distance function from a point $d(\cdot, p)$ is convex if and only if $p$ is a pole.

The following result is a generalization of the famous result of (Frankel 1966) about the fundamental group of positively curved manifolds. We remark that in our case the fundamental group $\pi_{1}(S)$ can be infinite, since the curvature of $S$ can be negative. We say that the Ricci radial curvature $\operatorname{Ric}_{S} \geq c$ when for any geodesic (not necessarily minimizing) $\gamma$ with $\gamma^{\prime}(0) \in v(S)$ it holds that $\operatorname{Ric}\left(\gamma^{\prime}, \gamma^{\prime}\right) \geq c$.

Theorem E. Assume that $S$ is a compact minimal hypersurface in $M$ and that $\mathrm{Ric}_{S}>0$ (or instead $\operatorname{Ric}_{S}^{\min }>0$ and $\operatorname{Ric}_{S} \geq 0$ ). Then the natural homomorphism of fundamental groups $\pi_{1}(S) \rightarrow \pi_{1}(M)$ is onto. 


\section{OUTLINE OF THE PROOFS}

Let $M$ be a Riemannian manifold of dimension $n$. Let $\gamma: \mathbb{R} \rightarrow M$ be a geodesic without conjugate points. Consider the normal bundle $v(\mathbb{R})$ associated with the isometric immersion $\gamma$. Take a closed neighborhood $U$ of $\mathbb{R}$ in $v(\mathbb{R})$ such that the normal exponential map $\exp ^{\perp}: U \rightarrow M$ is a local diffeomorphism. We consider in $U$ the Riemannian metric induced by $\exp ^{\perp}$. Let $\tilde{\gamma}: \mathbb{R} \rightarrow \mathbb{R}$ be the trivial curve given by $\tilde{\gamma}(t)=t$. We define the distance functions as

$$
r_{s}(x):=\operatorname{dist}(x, \tilde{\gamma}(s)), s \in \mathbb{R}, x \in U,
$$

where dist is the intrinsic distance in $U$. Since there are no conjugate points in $\tilde{\gamma}$, for sufficiently small $U$ we have that $r_{s}(x)$ is smooth at $x=\tilde{\gamma}(t)$ and $r_{s}(\tilde{\gamma}(t))=|s-t|$, for any $t \in(-\infty,+\infty)$. Also it is easy to see that $\nabla r_{s}(\tilde{\gamma}(t))=\tilde{\gamma}^{\prime}(t)$, for all $t>s$. For any unit vector field $X(t)$ which is parallel along $\tilde{\gamma}$, consider the Hessian $w_{s}(t):=\operatorname{Hess} r_{s}(\tilde{\gamma}(t))(X, X)$. Given some curve $c(u)$ with $c(0)=\tilde{\gamma}(t)$ and $c^{\prime}(0)=X(t)$, for small $|u|$ we have geodesics $\tilde{\gamma}_{u}$ joining $\tilde{\gamma}(s)$ and $c(u)$ with $\nabla r_{s}(c(u))=\tilde{\gamma}_{u}^{\prime}$. So we have $\nabla_{X} \nabla_{\nabla r_{s}} \nabla r_{s}=0$. Thus a direct calculation shows that

$$
\frac{d}{d t} w_{s}(t)=-K\left(X, \nabla r_{s}\right)-w_{s}^{2}(t)
$$

Lemma 1.1. With the notations above $w(t):=\lim _{s \rightarrow+\infty} w_{s}(t)$ exists for every $t$. The derivative $w^{\prime}(t)$ exists and $w^{\prime}(t)=\lim _{s \rightarrow+\infty} w_{s}^{\prime}(t)$.

To prove Theorem A we need the following lemmas, which are stated here without proofs. For a positive constant $a$, consider the following inequality of Ricatti type:

$$
\left\{\begin{array}{l}
x^{\prime}(t)+a x^{2}(t)+K(t) \leq 0 \\
x\left(t_{0}\right)=x_{0}
\end{array}\right.
$$

Lemma 1.2. If $\liminf \operatorname{in}_{t \rightarrow+\infty} \int_{t_{0}}^{t} K(s) d s \geq c$ for a constant $c \in R$ and $x(t)$ is a solution of (1.2) on $\left[t_{0},+\infty\right)$, then $\int_{t_{0}}^{\infty} x^{2}(s) d s<+\infty$.

Lemma 1.3. If $x(t)$ is a solution of (1.2) for $t \in \mathbb{R}$, then

$$
\xi:=\liminf _{\substack{u \rightarrow-\infty \\ v \rightarrow+\infty}} \int_{u}^{v} K(s) d s \leq 0 .
$$

We have $\xi=0$ if and only if $K(t) \equiv 0$ and $x(t) \equiv 0$.

Now we are in the position to prove our theorem.

Proof of Theorem A. It follows from (1.1) and Lemma 1.1 that

$$
w^{\prime}(t)+w^{2}(t)+K\left(X(t), \gamma^{\prime}(t)\right)=0, \text { for all } t \in \mathbb{R} .
$$

Therefore it follows immediately from Lemma 1.3 that $\eta \leq 0$ and that $\eta=0$ if and only if $K\left(X(t), \gamma^{\prime}(t)\right) \equiv 0$. 
Take an arbitrary $v$ with $|v|=1$ which is orthogonal to $\gamma^{\prime}\left(t_{0}\right)$. Consider an orthonormal basis $v=e_{1}, e_{2}, \cdots, e_{n}=\gamma^{\prime}\left(t_{0}\right)$, and the parallel transport $e_{i}(t), i=1,2, \cdots, n$ of $e_{i}$ along $\gamma$. For each $i=1, \cdots, n-1$ consider the sectional curvature $K_{i}(t)=K\left(e_{i}(t), \gamma^{\prime}(t)\right)$. Then we have the existence of functions $w_{i}: \mathbb{R} \rightarrow \mathbb{R}$ such that

$$
w_{i}^{\prime}(t)+w_{i}^{2}(t)+K_{i}(t)=0, \text { for all } t \in \mathbb{R}, \quad i=1, \cdots, n-1 .
$$

Set $y(t)=\sum_{i=1}^{n-1} w_{i}(t)$. Using the inequality

$$
\sum_{i=1}^{n-1} w_{i}^{2}(t) \geq \frac{1}{n-1}\left(\sum_{i=1}^{n-1} w_{i}(t)\right)^{2}
$$

we get

$$
y^{\prime}(t)+\frac{1}{n-1} y^{2}(t)+\operatorname{Ric}\left(\gamma^{\prime}(t), \gamma^{\prime}(t)\right) \leq 0, \text { for all } t \in \mathbb{R} .
$$

Therefore by Lemma 1.3 we have $\mu \leq 0$ and that $\mu=0$ if and only if $y(t) \equiv 0$ and $\operatorname{Ric}\left(\gamma^{\prime}(t), \gamma^{\prime}(t)\right) \equiv 0$.

From (1.5) we have

$$
y^{\prime}(t)+\sum_{i=1}^{n-1} w_{i}^{2}(t)+\operatorname{Ric}\left(\gamma^{\prime}(t), \gamma^{\prime}(t)\right) \equiv 0 .
$$

Since $y^{\prime}(t) \equiv 0$ and $\operatorname{Ric}\left(\gamma^{\prime}(t), \gamma^{\prime}(t)\right) \equiv 0$, we have from (1.7) that $w_{i}(t) \equiv 0$ for all $i=$ $1, \cdots, n-1$. Using (1.5) again we conclude that $K_{i}(t) \equiv 0$, hence $K\left(v, \gamma^{\prime}\left(t_{0}\right)\right)=0$. Theorem A is proved.

To prove Theorem B we use the same idea and the following lemma.

LEMMA 1.4. The following boundary value problem

$$
\left\{\begin{array}{l}
f^{\prime \prime}(t)+K(t) f(t)=0 \\
f^{\prime}(0)=0, \quad f(b)=0
\end{array}\right.
$$

has no positive solution on $[0, b)$, if $0<b<R_{S}$ and $\mathcal{R} \mathcal{I} C_{S}^{\min }(x) \geq K(\rho(x))$, for any $x \in M$.

Sketch of the Proof of Theorem C. Take a sequence $q_{k}$ as above. Assume by contradiction that $\delta_{k} r_{k}^{2} \geq \eta$, for some constant $\eta>0$. Set $\ell_{k}=r_{k} / 10$. Let $\varepsilon>0$ be a constant such that

$$
\frac{\sin \varepsilon}{\cos ^{2} \varepsilon}<\frac{\eta}{200} \text {. }
$$

Let $t_{k}=F_{o}\left(q_{k}\right)$, where $F_{o}(x):=\lim _{t \rightarrow+\infty}\left(t-d\left(x, S_{t}\right)\right)$, where $S_{t}$ is the boundary of the ball $B_{o}(t)$. We can prove that there exists a point $\tilde{q}_{k} \in \partial C_{t_{k}}$, such that $d\left(q_{k}, \tilde{q}_{k}\right)=\ell_{k}$. Let $\tau_{k}$ be a minimal geodesic joining $q_{k}$ and $\tilde{q}_{k}$. Consider a minimal connection $\sigma_{s}$ between $\tau_{k}(s)$ and $\partial C_{\left(t_{k}+\ell_{k}\right)}$, for 
$s \in\left[0, \ell_{k}\right]$. Set $\theta(s)=\measuredangle\left(\tau_{k}^{\prime}(s), \sigma_{s}^{\prime}(0)\right)$ and $\varphi(s)=d\left(\tau_{k}(s), \partial C_{\left(t_{k}+\ell_{k}\right)}\right)$. It is not difficult to obtain that $\tau_{k}\left(\left[0, \ell_{k}\right]\right) \subset C_{t_{k}}$. After a series of lemmas we show that

$$
\varphi\left(\ell_{k}\right) \leq \varphi(0)-\ell_{k} \cos \theta(0)-\frac{\bar{d} \lambda \delta_{k}}{2} \ell_{k}^{2} .
$$

For sufficiently large $k$, we have $\pi / 2 \leq \theta(0)<\pi / 2+\varepsilon$, and $\pi / 2 \leq \pi-\theta\left(\ell_{k}\right)<\pi / 2+\varepsilon$. So we obtain $\lambda \geq \min \left\{\sin ^{2} \theta(0), \sin ^{2} \theta\left(\ell_{k}\right)\right\} \geq \cos ^{2} \varepsilon$, because of the monotonicity of the function $\theta(s)$. We obtain also that $-\cos \theta(0) \leq-\cos (\pi / 2+\varepsilon)=\sin \varepsilon$. Thus we conclude that

$$
\ell_{k} \leq \varphi\left(\ell_{k}\right) \leq \ell_{k}+\ell_{k} \sin \varepsilon-\frac{\ell_{k}^{3}\left(\cos ^{2} \varepsilon\right) \delta_{k}}{2}
$$

Replacing $\ell_{k}$ by its value we conclude that

$$
\frac{\sin \varepsilon}{\cos ^{2} \varepsilon} \geq \frac{\ell_{k}^{2} \delta}{2} \geq \frac{\eta}{200},
$$

which contradicts to the choice of $\varepsilon$ and proves Theorem $\mathrm{C}$.

Sketch of the Proof of Theorem D. We can reduce again the estimate of Hessian of the distance function to estimate of solutions of Ricatti equations.

Sketch of the Proof of Theorem E. For any nonzero element $[\eta] \in \pi_{1}(M)$, there is a representative loop $\xi$ with base $p \in S$. We can find a curve $\tilde{\xi}$ in $\tilde{M}$ such that both initial point $\tilde{\xi}_{0}$ and end point $\tilde{\xi}_{1}$ in $\pi^{-1}(p)$. If $\tilde{\xi}_{1}=\tilde{\xi}_{0}$ there is nothing to prove. If $\tilde{\xi}_{1} \neq \tilde{\xi}_{0}$ then there exists a curve $\tilde{v}$ in $\pi^{-1}(S)$ connecting $\tilde{\xi}_{0}$ and $\tilde{\xi}_{1}$ which is homotopic to $\tilde{\xi}$. So $\pi \circ \tilde{v}$ is a loop in $S$ and $[\pi \circ \tilde{v}]=[\eta]$. This proves our conclusion.

\section{ACKNOWLEDGMENTS}

The authors would like to thank professor Manfredo do Carmo for his encouragement and support, professor Fuquan Fang for useful remarks and professor Francesco Mercuri for pointing to us a mistake in a previous version of this paper. The second named author would like to thank IMPA and UFF for the hospitality, while the work was done. Finally we acknowledge the financial support provided by $\mathrm{CNPq}$.

\section{REFERENCES}

ABresch U. 1985. Lower curvature bounds, Toponogov's Theorem, and bounded topology. Ann scient Éc Norm Sup 4e série, t. 18, 651-670.

Ambrose W. 1957. A Theorem of Myers. Duke Math J 24: 345-348.

Cheeger J \& Gromoll D. 1972. On the structure of complete manifolds of nonnegative curvature. Annals of Mathematics 96(3): 413-443. 
Cohn-Vossen S. 1935. Kürzeste Wege und Totalkrümung auf Flächen. Composito Math 2: 63-133.

Eschenburg J-H. 1987. Comparison theorems and hypersurfaces. Manuscripta Math 59: 295-323.

FRANKEL T. 1966. On the fundamental group of a compact minimal submanifold. Ann Math 83: 68-73.

GreEN LW. 1958. A theorem of E. Hopf. Michigan Math J 5: 31-34.

Heintze E \& Karcher H. 1978. A general comparison theorem with applications to volume estimates for submanifolds. Ann Scient Éc Norm Sup $4^{\circ}$ série, t. 11, 451-470.

Huber A. 1957. On the subharmonic functions and differential geometry in the large. Comment Math Helv 32: 13-72.

KASUe A. 1988. A compactification of a manifold with asymptotically nonnegative curvature. Annales Scientifiques de L'École Normale Supérieure 21(4): 593-622.

KLingenberg W. 1963. Manifolds with restricted conjugate locus. Ann Math 78: 527-547.

LIANG Y \& ZHAN H. 1996. The geodesics without conjugate point on a complete manifold. Tensor (N.S.) 57: $325-327$.

Myers SB. 1941. Riemannian manifolds with positive mean curvature. Duke Math J 8: 401-404.

YAU ST. 1991. Open problems in geometry, Chern, a great geometer, International Press. 